\title{
A Managerial Perspective of Technological Cloud Paradigms: its Effects on Enterprise Business, Costs and Strategies
}

\author{
Marco Remondino \\ Department of Economics \\ University of Genova \\ Email: marco.remondino@economia.unige.it
}

\begin{abstract}
The purpose of this paper is to analyse the technological model of "cloud computing" as instrumental for Enterprise Management and Economics. This is done through concrete data research to identify, highlight and evaluate the benefits, opportunities rather than costs and potential limits and risks in adopting it, and also potential strategies based on these services. Cloud computing will be briefly analysed under the technical point of view and then it will be examined under the strategical and applicative perspective of enterprises and managers.
\end{abstract}

Keywords - managerial perspectives, technological cloud paradigm, effects on enterprise, business, costs, strategies

\section{INTRODUCTION}

The scenario of the technological world and its natural and increasingly rapid evolution provide evidence that the classic vision of Information and Communication Technology (ICT) in the enterprise is to be significantly and continuously reviewed and updated in order to maintain or acquire strategic competitiveness.

Both end users and businesses, large or small, now have the possibility to enjoy data in a profoundly different way than before; the trend is in fact to move data and information to large Data Centres, located on the territory and use, for access and management of the same, remote services, with possible advantages in terms of costs of management and efficiency of the service.

Following the spread of so-called Web 2.0 and services for sharing and publishing content of any kind (think, as common examples and now part of the collective imagination, on YouTube, Myspace, and other services whose trend of popularity is constantly growing) has created the feeling in the user of being able to deploy advanced services without having to run into the classic problems of management of local resources (including installation difficulties, demand for computing and storage capacity, rapid obsolescence of applications and hardware). If this is valid for the common user, also the enterprises, whether they are large or small, from the strategic point of view, of cost-holding and the pursuit of efficiency and effectiveness objectives, have been facing many problems related to the management of large Data Processing Centres, among which:

- fleet management, in particular client machines

- rationalisation and optimisation of spaces

- rationalisation of electrical power
- strategic management of technological change (hardware and software)

The result is a gradual return to operational centralized philosophies and strategies, where the neuralgic system resides not on a mainframe or local server, owned by the company, or on a personal computer, but on one or more remote servers, managed by specific and well-identified service providers. The concrete possibility of a sort of outsourcing of those ICT services and infrastructures, previously managed internally by companies, is therefore configured, where the concepts of "operative elasticity" and efficiency are often rewarding.

It is common, in fact, that companies are forced to size their technological infrastructures according to the operational peaks (very intense, but limited to relatively short periods, generally for stationary or for markets) and not according to the average loads. This brings a potential waste of resources in the medium term and even more so in long-term periodic cycles.

These requirements, together with other strategic and contingent needs, have provided the basis for the definition of alternative paradigms for the management of data and information, first with Grid Computing and more recently with what, in some ways, is seen as its natural evolution: Cloud Computing.

All this is grafted onto the conception of an enterprise as a "complex social system": as we read in Paoli (2006), the complexity of the organization concerns the nature of the social relations through which the emergence of knowledge (which is necessary to innovate) is pursued and obtained. It follows that the control of innovation, in the broad sense, is increasingly controlling the evolution of systems and the underlying knowledge. Innovation is therefore seen as a strong strategic argument, which has a substantial impact on the organisation of the company. 


\section{THE BASICS OF CLOUD}

To understand the differences in strategic and applicative optics that concern this model, it is necessary to analyse the concept of "cloud" from a business perspective. The same will be examined in the following from different points of view, more markedly: technological and strategical.

In the first approximation (Weinmann, 2016) we can define the "cloud" model as a set of technologies and services based on virtualization, implemented by global providers. In particular, it's a model of use of computational resources or computer technologies (computing) through the Internet, that is, through the cloud. The details of the underlying infrastructure and architectural technologies are not known to users; actually they do not need to be known. Cloud computing therefore creates an abstraction of resources and makes them available to users, according to this new approach that transforms IT infrastructures into a service.

The term cloud computing, or simply cloud, refers to a set of technologies and methods of using computer services that favour the use and delivery of software, the ability to store and process large amounts of information over the Internet. The cloud provides, when needed, data transfer, retention or processing from users' computers to the provider's systems and vice versa. The cloud also allows you to take advantage of complex services without having to be equipped with computers and other hardware and without having a staff able to program or manage the system. Everything can be outsourced, at a potentially limited cost, as the IT resources needed for the services required can be shared with other parties which share the same needs.

Vaquero's research (2009) led the author to define the cloud as a "technological pool of virtualized resources that are easy to use and access. Cloud computing is often referred to not as a technology, but as a "business model", based on a number of technologies. Also, the term cloud computing is a metaphor for the computing resources (hardware and software) that companies and users access without the need to know exactly where hardware and software are physically located.

The International Data Corporation (IDC) distinguishes between cloud services and cloud computing: it defines cloud services as both business and consumer services that people use on the Internet, while cloud computing as an emerging development of IT.

Cloud computing allows you to use your data and applications anywhere and without space or time constraints, provided that you have an active internet connection. It is precisely this mobile aspect and the convenience of remote access to one's own resources that seems to be the driving force behind the choice of cloud solutions. About $59 \%$ of CIOs and $53 \%$ of IT managers believe that the application as a service approach is the main motivation that has driven them to approach cloud computing.

Moreover, economic leverage represents a strong push factor: Vaquero (2009) points out that $47 \%$ of the interviewees are attracted by the savings on infrastructure costs linked to the choice of virtualization strategies, while $42 \%$ are more motivated by the re-engineering of operating costs.

Among the distinctive features of cloud computing (Liu, 2013) are flexibility and scalability: if necessary, the resources allocated can be increased (or decreased) to have more or less bandwidth and performance. This factor affects $36 \%$ of respondents, but is more significant for the CIO target ( $42 \%$ compared to $28 \%$ for IT managers). As to the main drawback, cloud services and infrastructures are not yet perceived as totally secure: only $21 \%$ of respondents choose this technology because they believe it is safer or equally safe than traditional non cloud technologies. In figures 1 and 2, two graphs are shown, depicting the cloud adoption within big (with over 100 employees) and medium/small enterprises.
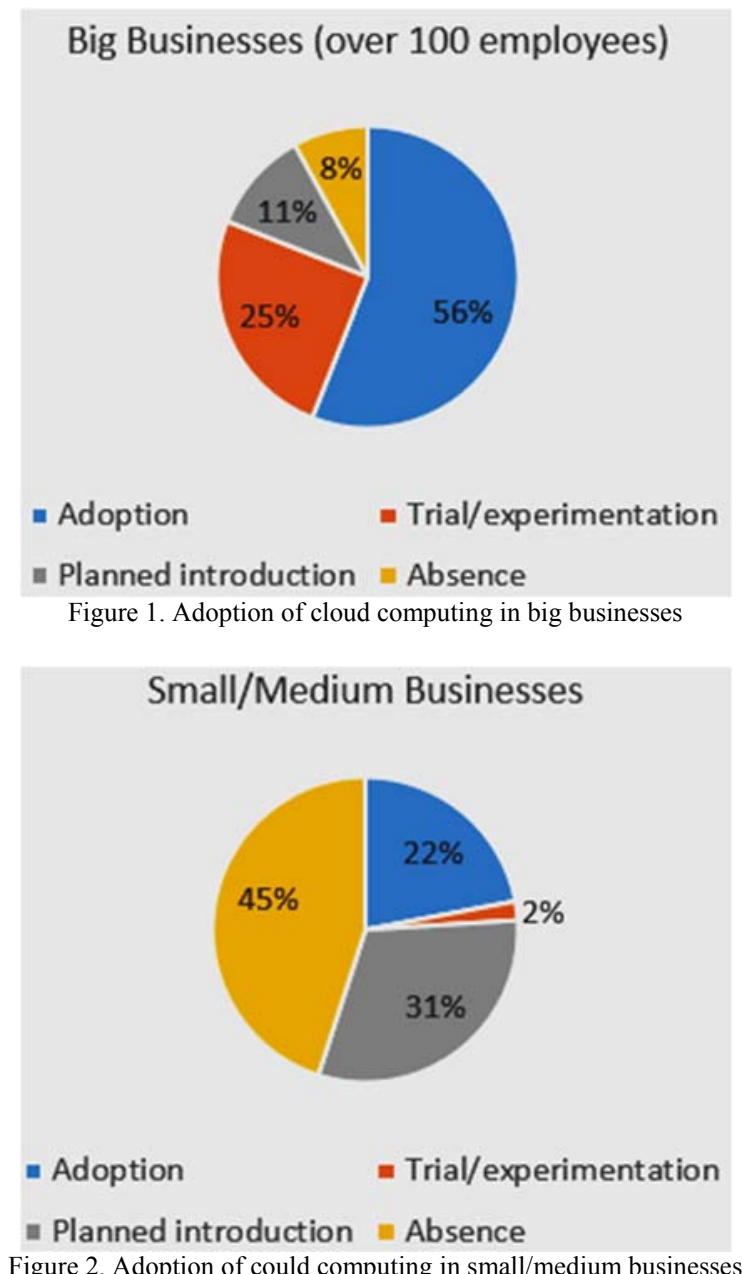

Figure 2. Adoption of could computing in small/medium businesses 
Paoli (2006) notes that it is necessary not to confuse instruments of circulation of significant information from mere company information systems, even though the word "knowledge" often occurs in these systems. The cloud, if used wisely by companies, can therefore be part of the first category, allowing a radically different way of obtaining data and information and to process the same. It is also necessary to distinguish between a real and integral ICT implementation, seen as opposed to implementation for the purpose of pure experimentation.

Among the supporters of the integral implementation and those of the implementation for the sole purpose of research through pilot tests, many now seem to converge on the idea that, to date, it is better to implement immediately everything that is not critical for the enterprise, while developing, at the same time, pilot tests to verify the reliability of various cloud solutions for future implementations even in critical areas.

This is what emerges from the research of Havanade, a company born out of the partnership between Accenture and Microsoft. In one of their documents, they identify as possible low-risk some back office activities or basic productivity applications such as email, CRM or human resources combined with the Software as a Service (SaaS) cloud. He recommends a move to Infrastructure as a Service (IaaS) if the company has already virtualized more than $40 \%$ of IT resources. An initial assessment of business objectives and IT portfolio will help companies formulate an appropriate cloud solution. In addition, it will be possible to find out at this point whether a pilot project is worthwhile or not (usually yes if the scale of the change is significant). If the pilot project is successful then the selected applications will be migrated from existing systems to the cloud, then the cloud will be managed, then the cloud will be managed to ensure a robust, highavailability environment. In table 1 , a survey depicting the diffusion of specific cloud application in the public sector is shown.

TABLE 1. PUBLIC CLOUD ADOPTION, 2015 AND 2016

\begin{tabular}{|c|c|c|}
\hline Running application & $\mathbf{2 0 1 5}$ & $\mathbf{2 0 1 6}$ \\
\hline AWS & $57 \%$ & $57 \%$ \\
\hline Azure IaaS & $12 \%$ & $17 \%$ \\
\hline Azure PaaS & $9 \%$ & $13 \%$ \\
\hline Vmware vCloud Air & $5 \%$ & $7 \%$ \\
\hline Google App Engine (PaaS) & $8 \%$ & $7 \%$ \\
\hline IBM SoftLayer & $5 \%$ & $7 \%$ \\
\hline Google IaaS & $5 \%$ & $6 \%$ \\
\hline DigitalOcean & na & $5 \%$ \\
\hline Oracle Cloud (IaaS) & na & $4 \%$ \\
\hline
\end{tabular}

Dell identifies two paths to the cloud: an evolutionary one, based on virtualization and infrastructure oriented
(IaaS). A revolutionary platform-based (Paas) and application oriented (SaaS) one, where scalability and redundancy are integrated. Dell's enterprise cloud deployment scenario predicts that private, public and hybrid cloud computing models will not always replace conventional or virtualized IT models, but in many cases will complement them.

While security and privacy are perceived problems that are gradually being solved with a gradual increase in trust by companies, the reduced control can, at least partially, solve with an adequate architecture and an adequate negotiation between supplier and user, the reduced preparation of human resources through training; the problem of the network is structural. Without an adequate internet network, the cloud cannot exist; this is why this braking factor deserves particular attention.

\section{BENEFITS AND RISKS FOR ENTERPRISES}

Cloud providers offer customers affordable accessibility (such as on-demand and pay-per-use services). Enterprises can save on IT costs by paying only for what they have actually used; they do not need to invest in hardware, software, networks and IT staff. The network security service is also managed by cloud computing providers. Another important advantage of moving to the cloud is the scalability and flexibility of the IT that cloud computing providers offer. Cloud computing users can enjoy the flexibility of changing workload, especially managing the increase in commercial transactions during the hot season. Users who move to cloud computing just like outsourcing their IT operations to suppliers. For this reason, users do not need to manage IT tasks within the organisation. Another advantage of switching to the cloud is that of improving mobility: customers can access the software from anywhere, as long as access to the Internet is feasible.

The main risks related to cloud computing technologies are in the following areas: authentication, data security and privacy, interfacing with internal systems, system availability, business continuity and ownership of content and other legal requirements (Raval, 2010).

Among these, security and privacy are the areas which are most affected since, in cloud computing model, data are stored outside the organization (Dutta et al., 2013). In cloud computing practices, customers (or business users) outsource servers and server applications, data storage, and software programs from a cloud service provider (or providers) to receive on-demand and pay-per-use services. Most cloud service providers offer a measurement service - meaning that they charge customers for the processing capacity that customers have actually used. This special arrangement makes the audit work of cloud computing different from the traditional IT control. It is recognized that information deliver value to user organizations and to businesses. Table 2 summarizes these concepts. 
TABLE 2. SUMMARIZATION OF BENEFITS AND RISKS OF CLOUD ADOPTION WITHIN ENTERPRISES

\begin{tabular}{|l|l|}
\hline \multicolumn{1}{|c|}{ Benefits } & \multicolumn{1}{c|}{ Risks } \\
\hline Accessibility & $\begin{array}{l}\text { Authentication and data } \\
\text { security/privacy }\end{array}$ \\
\hline Cost saving & $\begin{array}{l}\text { Interfacing with existing internal } \\
\text { systems and business continuity }\end{array}$ \\
\hline $\begin{array}{l}\text { Scalability and } \\
\text { flexibility }\end{array}$ & System availability \\
\hline Mobility & $\begin{array}{l}\text { Ownership of content and other legal } \\
\text { issues }\end{array}$ \\
\hline
\end{tabular}

\section{CLOUD FOR INTERNATIONALIZATION AND COLLABORATION OF ENTERPRISES}

Cloud computing appears as a facilitator of the internationalization of enterprises, as it eliminates the need to transport computers and allows people to always have available applications and files anywhere in the world and updated in real time (or almost, depending on the bandwidth of the Internet connection), thus allowing a closer collaboration between business resources, regardless of the physical-geographical distance.

International collaboration via cloud, in addition to all the issues related to international collaboration in general, must also address the issues related to the dematerialization of collaboration. From a sociological point of view, it could generate problems and resistance from human resources, which in a borderline scenario would be in a room, alone with a computer (or any other mean for accessing the network), rather than in an environment specifically dedicated to the team and physically inhabited by the members of the team. Therefore, it is necessary to train human resources and to be aware of the advantages and disadvantages of the virtual collaboration environment, so that they are able to take advantage of the advantages, but above all to solve the disadvantages, as it would be impossible for the Governance to intervene personally, at competitive costs, to facilitate the resolution of problems, given the international distances that may be present between the members of the team. It is very suitable for high profile individuals and legal entities (e.g. partnerships, joint ventures, mergers and acquisitions, etc.). The cloud therefore allows internationalization with shared standards also in terms of applications and working methods, starting from the sharing of information technologies via the Internet. Several companies have offerings for the cloud market, focusing on four areas: People, Finance, Customers and Suppliers.

In Paoli, 2006, we read that products and production processes are artefacts that can no longer be considered objects, but real systems, whose evolutionary dynamics are the result of a complex interweaving of processes that unfold in the system (often at all hierarchical levels and simultaneously, as noted ta Takeishi and Fujimoto, 2003). In his research, Paoli also notes that in the presence of innovation or organizational change there is a flow of changes in the rules and methods of interaction between evolutions of the structures/functions of the system's components. This may be due to the diversity of the paradigms of reference and the nature of the knowledge bases on which they are based. It seems to me that the cloud, as a model for a company's collaborative strategy, can be rightly included in this category of innovation and should be treated as such.

An excellent cloud strategy, can and perhaps should just start from the inside and then transfer the IT in cloud from the inside to the outside, being able to choose the best services in outsourcing, but after having matured internally to the enterprise or the country already a remarkable experience.

Intel, a well-known company in the IT market, acts in this way, and in one of its white papers it illustrates in general terms how it uses and implements cloud computing and the philosophy of using this technology, proven by encouraging and significant results.

An internal cloud implementation also lays the foundation for easier outsourcing that could take place in less time and in a much more natural way. Intel writes: "We will initially grow our internal virtualized computing environment to support growth in cloud type attributes over time. We plan to aggressively expand and evolve this internal environment. Intel's goal is to migrate selectively to external clouds as suppliers are able to deliver mature services, barriers to adoption in the enterprise are overcome, and opportunities arise to improve flexibility and agility as well as cost reduction. This is because according to Intel, and according to this research that has found the phenomenon several times and on more companies, several concerns stop an adoption by external cloud companies, such as security risks and the lack of a mature technology and standards.

Intel's internal cloud method also aims to drain experience from various cloud providers to deploy solutions captured in the marketplace internally. In fact, about SaaS Intel states: "SaaS was used as a temporary selection while Intel developed in-house solutions" or "this approach has enabled us to take advantage of the experience of suppliers".

Intel provides a case by case scheme that could be generally valid in all situations where there were both internal and external clouds (see Figure 3). 


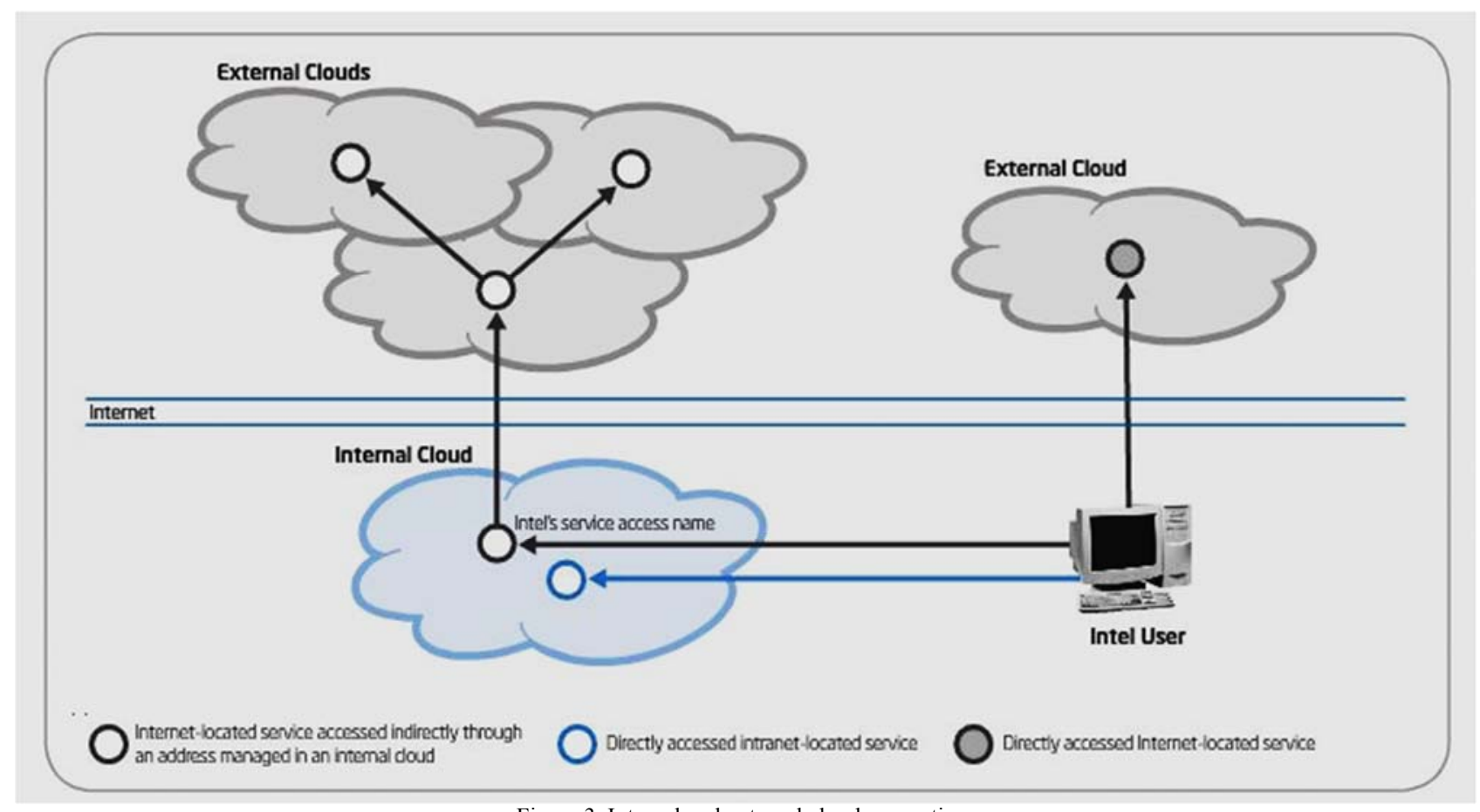

Figure 3. Internal and external cloud computing.

Future IT organizations will be responsible for identifying the right "location" for cloud computing across the different platforms available, some within the enterprise and others externally, and for evaluating the different global cloud providers and bringing together offerings in a right mix internally and externally.

It is likely that many companies will follow the hybrid model. In such a scenario, collaboration among companies will be privileged at local or national level as they will be able to access all IT resources through the internal network, while only in a later phase full collaboration will be available at international level. This scenario therefore includes significant benefits for the local/internal dimension at least for a certain period of time, exactly what is needed for a complete transition of enterprise IT into external clouds, including the most critical applications.

It should be stressed that from the point of view of internal collaboration, the cloud can generate many cases of tension. The "Delivering on High Cloud Expectations" survey conducted by Forrester Consulting on behalf of BMC Software shows that $72 \%$ of the CIO, of the sample of 327 IT managers and architects in the US, Europe and Asia-Pacific, agree that executives see the cloud as a way to be IT-independent. $58 \%$ of respondents perform mission-critical workloads on unmanaged public cloud services, while only $36 \%$ rely on policies that allow this. A collaborative meeting point, which emerges from the survey data, is $71 \%$ of respondents who think IT should be responsible for ensuring that public cloud services meet business requirements for performance, security and availability.

\section{VALUE CREATION}

Chou (2015) notice that "Valuation is an economic process that identifies the value of actions in an organization. Value plays an important role while organizations are determining an action-taking arrangement. While business executives intend to invest in a vital project, they must measure the possible outcome of satisfaction in that venture. Value is therefore a foundation of decision-making. Without a doubt, IT delivers value to organizations. IT generates value by utilizing computing power in the work place, in their time saving, cost deduction, and decision-support function can be achieved is a vivid indication of IT value realization".

That said, it is useful to think about which kind of value each IT technology is able to create within an enterprise. The value chain is a model that allows the structure of an organisation to be described as a limited set of processes. This model was theorized by Michael Porter in 1985 in his well-known book: "Competitive Advantage: Creating and Sustaining Superior Performance". Cost trends can be better understood by disaggregating the company into its strategically relevant activities. To this end, Porter proposed the value chain tool (value is here the sum that buyers are willing to pay for what a company provides them). In order to examine the areas of intervention for the creation of a competitive advantage, in the Porter scheme 
the company is disaggregated into nine interdependent activities, of which five are primary and four support activities.

- Primary activities: 1) Inbound logistics; 2) Operational activities; 3) Outbound logistics; 4) Marketing and sales; 5) Services.

- Support activities: 1) infrastructure activities; 2) human resources management; 3) technology development; 4) procurement.

The primary activities are those related to the physical creation of the product, its marketing and delivery to buyers and after-sales service. Support activities provide the inputs and infrastructure to enable the primary activities to be carried out. Each activity requires the use of purchased equipment, human resources and different combinations of technologies. The respective costs are linked to each activity, the structure of which is highlighted.

The acquisition of the necessary inputs comes essentially from outside; only a small part comes from internal support activities. Technology and its development control, albeit with different intensity, the entire production process, and not a single activity. Human resource management is also characterised by its presence in all activities. Finally, infrastructure activities include other functions that cover the entire company, including general management, finance, management control, legal services, and external relations. Value-creating assets are linked in the sense that the way an asset is carried out affects the cost or effectiveness of another asset. For example, more accurate and therefore more expensive product design can reduce after-sales service costs.

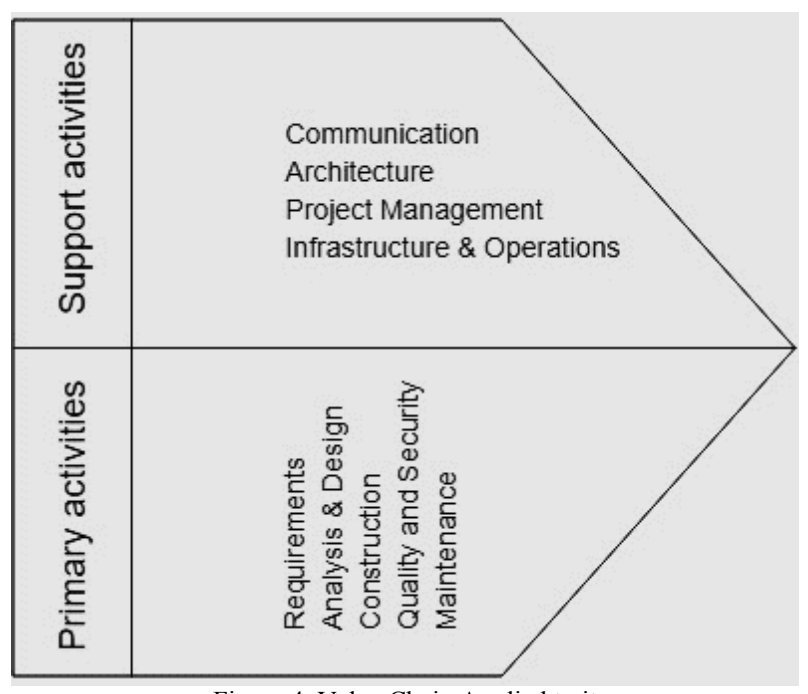

Figure 4. Value Chain Applied to it

The identification of the activities that make up the enterprise is the first step of the analysis. The related costs and revenues are linked to the individual activities, so as to highlight possible areas for improvement and allow targeted interventions.
The concepts of value chain can be applied to IT, as shown in figure 4.

For the cloud computing, it is evident (Kulluro, 2013) how the support activity become fundamental for the enterprise that is willing to adopt it. In particular, they need to choose the right architecture (platform) for their specific needs, and they have to make the infrastructure the more compatible with existing IT systems already present within the enterprise. Besides, the project management activity is also crucial in defining exactly which processes will have to become cloud based and according to which models. Finally, communication plays a strategic role, as seen before in the paragraph devoted to enterprise collaboration, since cloud helps in both communicating among distance subjects and in team working.

On the other side, some of the primary activities are demanded to the cloud providers (and this is something potentially scaring for the enterprises, as seen in the paragraph devoted to benefits and risks). In particular, the activity regarding quality and security is crucial for IT and, in cloud computing, it is managed by the external provider. The same goes for the "construction" activity; since we are dealing with a SaaS or even a IaaS paradigm, it's not the internal IT department that builds the platform. Here, it is important rather to be able to configure and customize it as much as possible, as if the resulting platform is the most respondent as possible to the needs of the company. For this reason, the "requirements" activity/phase is crucial. The IT management and governance within a company needs to have a very precise overview of what the enterprise requires. Only in this way they'll be able to interface themselves with the personnel of the cloud provider, and ask for a tailored service to satisfy their needs. Analysis and Design activities, hence, have to be jointly performed both by the internal IT department of the customer enterprise and by the seller (provider) of cloud service. Maintenance activity is then totally outsourced, as already seen in the previous paragraph about benefits.

Summing up, it's evident that cloud model could, potentially, heavily impact the value creation chain for IT within an enterprise. Only by carefully managing all the involved activities this model can create value and leverage on those activities, often considered as "support activities" for traditional IT models, which become now strategic and, in a way, really fundamental, for the cloud model.

\section{COSTS: CAPEX VS OPEX}

Commonly, the expenses for IT fall under "capital expenses", usually referred to as CapEx. From what has been written in the previous paragraphs, it is immediately evident that cloud computing moves this conception. The "as Service" model, be it applied to software or to the whole architecture, make it clear that now the IT expenses are shifting to the field of "operating expenses", or OpEx. 
Capital expenses are generally and traditionally considered as investments for the enterprise, meaning that the company is acquiring something which becomes an asset and is clearly identifiable as "owned", after it has been bought. Some investments may be followed by the so called "maintenance agreements", but the main good is owned by the enterprise. An example can be buying a certain number of personal computers or mainframes and then stipulating a contract for their maintenance and constant upgrading overtime. The same applies to software, which can be bought once (again by using the CapEx model) and then constantly upgraded with further contracts.

OpEx are very different compared with CapEx, in that an operational expenditure is an ongoing cost, recurring with regularity (typical examples are periodic bills). The costs belonging to this specific category are usually lower than those in CapEx field, but they recur regularly and hence it should be considered the whole period, for an appropriate comparison. From the point of view of an IT manager, it could be easier to get approval for apparently lower, repeated OpEx than for huge "one shot" CapEx. Besides, for temporary projects, buying expensive infrastructures would not make sense, making renting the best viable option.

Besides, with OpEx what is actually used by the enterprise is more evident. For example, we can think to "storage as a service". Instead of using local disks, enterprises can "rent" disk space. They'll know exactly how much space they were renting in a certain period and how much they were paying for it. With CapEx, it is sometimes difficult to detail how a company benefits (directly and indirectly) from specific investments.

Cloud computing clearly marks a shift from CapEx to OpEx for many specific IT related expenses. Storing data in its own Data Centre requires a capital expense from the enterprise, i.e. to bear the cost of developing or providing durable assets for the product or system. On the contrary, using an external cloud service that offers pay-as-you-go services represents an "Operating Expense", and the cost necessary to manage a product, business or system is fractioned on a "per month" basis and above all only targeted to what is really needed.

The topic inevitably leads to positive comparisons and there have been discussions in matching the cost of a service such as Amazon EC2 (Amazon Elastic Compute Cloud is a web service that provides to ensure the computing capacity in the cloud) with the cost of a server within the company's data centre. Usually the average selling price of a server is considered and divided by 36 (the number of months of typical expected service life for a hardware appliance). Therefore, it is suggested that Cloud Computing is more expensive than owning a server in a corporate Data Centre, which means that it is inadequate for use in typical business applications that require daily availability.
To give more value to the concept, we can compare the real cost of the internal data centre vs external Cloud. Comparing the monthly cost of an EC2 server to a similar one in a data centre doesn't take into account:

1. direct costs associated with server activation: power, space, storage and IT operations for managing these resources.

2. indirect costs of operating a server: network, storage facilities and IT operations to manage general infrastructure.

3. The high costs of a server: procurement and accounting staff, not to mention the loss of critical resources: IT management and its assistance.

When you add these factors to the cost of an internal server, the total monthly cost of a proprietary server increases significantly. In a recent UC Berkeley Paper, the RAD Lab estimated that cloud providers have very competitive costs: $75-80 \%$ less than an internal Data Centre. Some of these advantages are due to purchasing power through volumes, some due to more efficient management because, as the mentioned research shows, these companies are managed as profitable companies with a strong focus on costs.

Therefore, the debate about the cost of a typical data centre and cloud provider costs is usually too simplified and it's not possible to have a true cost structure for a realistic comparison. This should not come as a surprise, as most IT organizations do not really have a clear understanding of their real costs or the models to adopt (e.g. an "Activity-based cost" approach). Another point of view on why this comparison falls comes from a blog post by Steven Oberlin, Chief Scientist of Cassatt, who observed that in this type of cost comparison the use of the internal server is ignored: if for example it is turning to $20 \%$ of its potential, the actual cost of a given level of processing is actually five times higher than that normally assumed in these cost comparisons.

Despite the IT organization's motivation to keep its own Data Centre alive, the General Management seems interested in Cloud Computing. This is the crucial point that begins to generate interest in moving the cost view from CapEx to OpEx. The author cites his own direct experience and not a positive one. He records how there is still a lack of analytical attitude in defining, when comparing the two technologies, or the two types of expenditure and hearing the importance of the relative one of Cloud Computing, because everything is based on cash flow and if the expenditure is for a capital asset or payment for an installation of EC2, it is always a question of equal amount of money. In doing so, we are not dealing with the subject we have just discussed, namely we are not talking about the same amount of money, but about achieving a specific goal. The statement is that, given a certain amount of money to be paid, the instalment becomes irrelevant. These and other considerations are summed up in the following table 3 . 
TABLE 3. COMPARISON OF DIFFERENT IT ENTERPRISE STRATEGIES

\begin{tabular}{|c|c|c|c|}
\hline & Purchased IT & Managed IT & Cloud \\
\hline Procurement times & Weeks/months & Days/weeks & Minutes/hours \\
\hline $\begin{array}{l}\text { Scalability of } \\
\text { infrastructure }\end{array}$ & $\begin{array}{l}\text { Usually expensive } \\
\text { Potentially limited }\end{array}$ & $\begin{array}{l}\text { Relatively expensive } \\
\text { Potentially limited }\end{array}$ & $\begin{array}{l}\text { Pay per Use } \\
\text { Flexible }\end{array}$ \\
\hline Investments & High CapEx & OpEx with long contracts & Use based OpEx \\
\hline Environmental Impact & Normal or limited & Normal or limited & Limited \\
\hline Pricing model & $\begin{array}{l}\text { Purchase of infrastructures } \\
\text { (even when under used) }\end{array}$ & $\begin{array}{l}\text { Rental of equipment } \\
\text { (even when under used) }\end{array}$ & Use based \\
\hline Control over data & Very high & High & Medium/High \\
\hline Privacy & Very high & High & High \\
\hline Access rapidity & Instant access & Instant access & $\begin{array}{l}\text { Dependent on } \\
\text { connection }\end{array}$ \\
\hline
\end{tabular}

For a start, although the cash flow is the same, the choice of Cloud would be more attractive. This is because paying for capital goods like a server is a business commitment regardless of whether it is used or not. Once you have purchased a good, you are bound with it; it is like buying a car or any other substantial good. Conversely, if you rent a car, you are bound to its cost only for the period you want to use and once you have paid for that use, you have no other financial obligations. The most interesting aspect is that everyone more or less is aware that you pay a premium for this flexibility, that is, a rental car costs more per day than the same car, if purchased.

Consequently, even if the Cloud alternative is more expensive in a known period, it is easy to understand that, after the period necessary for the development of a project, the costs related to the use of the infrastructure are reduced and potentially tend to zero. Moreover, the value referred to the scalability offered by the cloud alternative is known (as depicted in table 2): the production activity could have moments of growth/decline (and with it the consumption for a short period of computing capacity could increase/decrease). The important element with cloud computing is that this involves the application of tariffs linked to the need for expansion/contraction, while an owned infrastructure cannot be changed so easily.

We can summarize, for the importance of the topic dealt with in this paragraph, that the provision of new services based on a cloud platform allow to obtain three different types of benefits:

- Move the budget's centre of gravity from CapEx to OpEx and consequently generate a close correlation among what is spent on the necessary resources and the trend of adoption (and economic return) of a service. It is immediately possible to evolve towards a way of using IT resources, including a pay-per-use approach.
- Allow the service owner to reduce (or not increase) the cost of personnel to administer an IT system when the company's purposes change

- Simplify service expansion because no major investment is required in Capex to expand or upgrade existing IT architecture to support new uses in the event of a successful service.

\section{CONCLUSION}

While cloud computing is now quite an established and accepted paradigm, as of beginning of 2017 it still is not widely diffused and adopted within small and medium enterprises. In the paper, a series of potential benefits is considered for which this model could be preferred to the traditional purchase of IT infrastructures or software.

First of all, it is important to deal with the change of perspective of the "as a service" paradigm. Both SaaS and IaaS deeply change the way in which both software and infrastructures are dealt with, inside companies. This cultural change involves releasing that with cloud computing the enterprise can actually pay for what it actually uses and consumes. Besides, cloud services allow enterprises to always have an up-to-date "system", whereas traditional paradigms involve also the necessity of purchasing IT services and licences.

Another important cultural change is the shifting from CapEx to OpEx, when dealing with cloud services. When IT infrastructures need to be bought, the expenses are typical CapEx, while the "as a Service" approach turn them into OpEx.

The main concerns about cloud paradigms seem to be security (Martin, 2014), privacy and control over data; even if these are understandable, above all when data are critical for enterprise and business strategy, recent advancements in cloud services have allowed a very high level of security, 
possibly higher than internal, but mismanaged, IT solutions.

In conclusion, while big enterprises have almost completely embraced some form of cloud services in the latest years, it's possible to foresee that also medium and small enterprise will soon transition towards these kind of services in the following few years.

\section{REFERENCES}

[1] Baldwin, C. and K. Clark (2000). Design Rules, Vol 1: The Power of Modularity. MIT Press.

[2] Baldwin, C. and Woodard. C.J. (2007) Competition in Modular Clusters. Harvard Business School working.

[3] Chou, D. C. (2015). Cloud computing: A value creation model, Comput. Stand. Interfaces, vol. 38, pp. 72-77.

[4] Cinquini L., Di Minin A., Varaldo R. (2011). Nuovi Modelli di Business e Creazione di Valore: la Scienza dei Servizi, Ed. Springer, ISBN 978-88-470-1844-00.

[5] Dutta, A., Chao Alex Peng, G. U. O., \& Choudhary, A. (2013). Risks in enterprise cloud computing: The perspective of IT experts. Journal of Computer Information Systems, 53(4), 39-48.

[6] Gawer, A. (ed) (2009), Platforms, Markets and Innovation, Cheltenham, UK and Northampton, MA, US

[7] Kolluru, N. V. S., \& Mantha, N. (2013). Cloud integration Strategy to connect applications to cloud. In 2013 Annual IEEE India Conference, INDICON 2013.

[8] Liu, H. (2013). Big data drives cloud adoption in enterprise. IEEE Internet Computing, 17(4), 68-71.

[9] Martin, J., \& Lodewijkx, K. (2014). Securing Cloud Environments for Enterprise Computing. In Proceedings of the 6th edition of the ACM Workshop on Cloud Computing Security - CCSW'14 (pp. $1-1)$.

[10] Paoli M. (2006). Management della Complessita, Complessita del Management, Ed.FrancoAngeli, ISBN 88-464-7898-3.

[11] Takeishi, A. and Fujimoto, T. (2003). Modularization in the Car Industry: Interlinked multiple hierarchies.

[12] Vaquero, Rodero-Merino, Caceres, Linder (2009). A Break in the Clouds: Towards a Cloud Definition".

[13] Weinman, J. (2016). The Economics and Strategy of Manufacturing and the Cloud. IEEE Cloud Computing, 3(4), 6-11. 